\title{
The interstitial lung disease spectrum under a uniform diagnostic algorithm: a retrospective study of 1,945 individuals
}

\author{
Bingpeng Guo ${ }^{1}$, Lulin Wang ${ }^{1}$, Shu Xia ${ }^{1}$, Mengmeng Mao ${ }^{1}$, Weiping Qian ${ }^{1}$, Xiaomin Peng ${ }^{1}$, Zexuan Zheng ${ }^{1}$, \\ Rongchang Chen ${ }^{1,2}$, Qian Han ${ }^{1}$, Qun Luo ${ }^{1}$ \\ ${ }^{1}$ State Key Laboratory of Respiratory Disease, National Clinical Research Center for Respiratory Disease, Guangzhou Institute of Respiratory \\ Health, The First Affiliated Hospital of Guangzhou Medical University, Guangzhou, China; ${ }^{2}$ Shenzhen Institute of Respiratory Diseases, Shenzhen \\ People's Hospital, Shenzhen, China \\ Contributions: (I) Conception and design: B Guo, Q Han, Q Luo; (II) Administrative support: R Chen; (III) Provision of study materials or patients: \\ Q Han, Q Luo; (IV) Collection and assembly of data: L Wang, S Xia, M Mao, W Qian, X Peng, Z Zheng; (V) Data analysis and interpretation: B \\ Guo, Q Han; (VI) Manuscript writing: All authors; (VII) Final approval of manuscript: All authors. \\ Correspondence to: Qian Han; Qun Luo. State Key Laboratory of Respiratory Disease, National Clinical Research Center for Respiratory Disease, \\ Guangzhou Institute of Respiratory Health, The First Affiliated Hospital of Guangzhou Medical University, No. 151 Yanjiang Road, Guangzhou \\ 510120, China. Email: hanqian1020@yahoo.com; luoqunx@163.com.
}

Background: Reported data on the disease spectrum of interstitial lung diseases (ILDs) of China are sparse and varied. We aimed to investigate the spectrum of ILDs and the distribution of diagnostic methods under a uniform diagnosis.

Methods: This retrospective study enrolled ILDs cases from Guangzhou Institute of Respiratory Health (GIRH). All cases were classified into specific subgroups of ILDs according to updated guidelines.

Results: A total of 1,945 subjects were enrolled from January 2012 to December 2017. The mean age was 57.9 years, and 1,080 (55.5\%) patients were male. The most common subtype of ILDs was idiopathic pulmonary fibrosis (IPF, 20.3\%), followed by interstitial pneumonia with autoimmune features (IPAF, 17.9\%), connective tissue disease associated ILD (CTD-ILD, 18.3\%) and unclassifiable idiopathic interstitial pneumonia (UIIP, 14.7\%). A total of 818 (42.1\%) patients underwent lung biopsy in order to obtain a histological diagnose. TBLB was performed in 565 (29.0\%) patients, eleven of whom underwent SLB because TBLB failed to obtain lung samples. SLB was performed in $213(11.0 \%)$ patients and TBCB was performed in $51(2.6 \%)$ patients. Among them, histological results were considered clinically helpful in $722(88.3 \%)$ cases, and provided definitive histopathological diagnoses in 368 cases. Surgical lung biopsy (SLB) was performed in $213(10.9 \%)$ subjects, while $115(54.0 \%)$ cases were performed among the idiopathic interstitial pneumonia (IIP). Among SLB cases in IIP, the highest rate of SLB was desquamative interstitial pneumonia/ respiratory bronchiolitis-interstitial lung disease (DIP/RB-ILD, 10/10), lymphoid interstitial pneumonia (LIP, 9/9), followed by cryptogenic organizing (COP, 18/26), nonspecific interstitial pneumonia (NSIP, 22/53), IPF (43/395), UIIP (13/285).

Conclusions: IPF was the most common ILDs in our ILD center, followed by IPAF, CTD-ILD and UIIP. Histological information may help to establish diagnostic algorithm in ILD.

Keywords: Interstitial lung disease (ILD); epidemiology; surgical lung biopsy (SLB)

Submitted Dec 15, 2019. Accepted for publication Jun 02, 2020.

doi: $10.21037 /$ jtd-19-4021

View this article at: http://dx.doi.org/10.21037/jtd-19-4021

(C) Journal of Thoracic Disease. All rights reserved. 


\section{Introduction}

Interstitial lung disease (ILD) encompass a heterogeneous group of rare diseases that affect the pulmonary parenchyma with various degrees of pulmonary inflammation and fibrosis (1). Accurate diagnosis and classification of ILD are essential, while erroneous diagnosis result in adverse outcome in patients by means of inappropriate treatment. For example, administration of steroids or immunosuppressive agent in idiopathic pulmonary fibrosis (IPF) have been shown to be harmful (1), and the inappropriate initiation of antifibrotic drugs for diseases where the efficacy are unknown. However, the difficulties of making accurate and specific diagnosis in the ILD are limited by unavailable histopathology data and high interobserver variability $(2,3)$. Therefore, diagnostic approaches like multidisciplinary discussion (MDD) and surgical lung biopsy (SLB) in some given cases are endorsed by the international guidelines $(1,4,5)$. MDD resulted in a change in diagnosis in more than one-third of individuals, and those patients undergoing SLB were significantly more likely to receive a confident diagnosis compared with those without SLB (6). However, only limited studies reported the data of diagnostic approaches in accordance with recent guidelines of ILDs in real world.

Several studies have reported the incidence, prevalence and spectrum of subtype of ILD. The incidences of ILD are estimated between 4.6 to 31.5 per 100,000 in developed countries (Europe and North America) (7-10). The spectrum of ILD varies around the world because of different environmental factors, occupational exposure, sociocultural, economic practices, and health insurance policies (11). Connective tissue disease (CTD)-ILD, IPF, sarcoidosis and hypersensitivity pneumonia (HP) were the most frequent types of ILD in developing countries (India, Saudi Arabia and China) (11-13). However, most of studies have not used the term "interstitial pneumonia with autoimmune features" (IPAF) proposed by the 2015 European Respiratory Society (ERS)/American Thoracic Society (ATS) (14). Although the term IPAF is now of a provisional category of ILD, it unifies a criteria for many patients with idiopathic interstitial pneumonia (IIP) having subtle features suggesting an autoimmune etiology but not meeting the classification criteria for a specific CTD.

In this retrospective study, we collected the clinical data of patients diagnosed with ILD in the Guangzhou Institute of Respiratory Health (GIRH). Our study has two main aims: (I) to describe the pattern of diagnostic approaches in our center followed by recent guidelines of ILD in recent years; (II) to report the spectrum of ILD, which could provide more data to ILD epidemiology.

\section{Methods}

We retrospectively collected the medical records of 1,945 patients who were newly diagnosed with ILD in GIRH from January 2012 to December 2017. Since the statement of IPAF was proposed after July, 2015, two of the investigators (QL and $\mathrm{QH})$ reviewed the medical records and re-diagnosed patients who had been diagnosed with undifferentiated CTD-ILD and non-IPF IIP between January 2012 to July 2015.

The trial was conducted in accordance with the Declaration of Helsinki and the Harmonized Tripartite Guideline for Good Clinical Practice from the International Conference on Harmonization. The study was approved by the Institutional Human Ethics Review Committee in our institute (approval number2020 K-10). All patients enrolled completed the informed consent form.

\section{Diagnosis of ILD}

The diagnosis of IPF was followed by the ATS/ERS/Japanese Respiratory Society/Latin American Thoracic Association guidelines (1). For the diagnosis of IIPs, the ATS/ERS Multidisciplinary Consensus Classification of the IIPs was followed (4,15). The American College of Rheumatology criteria were used to establish the diagnosis of CTD (16-20). IPAF was diagnosed using the ATS/ERS statement (14). The diagnosis of HP was made based on the environmental or occupational exposures, and the presence of air-trapping/ mosaic attenuation on HRCT or biopsy showing rare giant cells, minor lymphocytic bronchiolitis, or airway-centered fibrosis (21). Sarcoidosis was diagnosed based on the statement of ATS/ERS/World Association of Sarcoidosis and Other Granulomatous Disorders (WASOG) (22).

\section{Diagnostic algorithm of ILD}

The diagnostic approach of ILDs in our center including the following steps: first, we took a detailed history of patients who were suspected of ILD by clinicians (presented with unexplained dyspnea on exertion, and/or cough, with/ without bibasilar inspiratory crackles). The detailed history questionnaire included identifiable causes of ILDs, such as domestic and occupational environmental exposures, CTD 


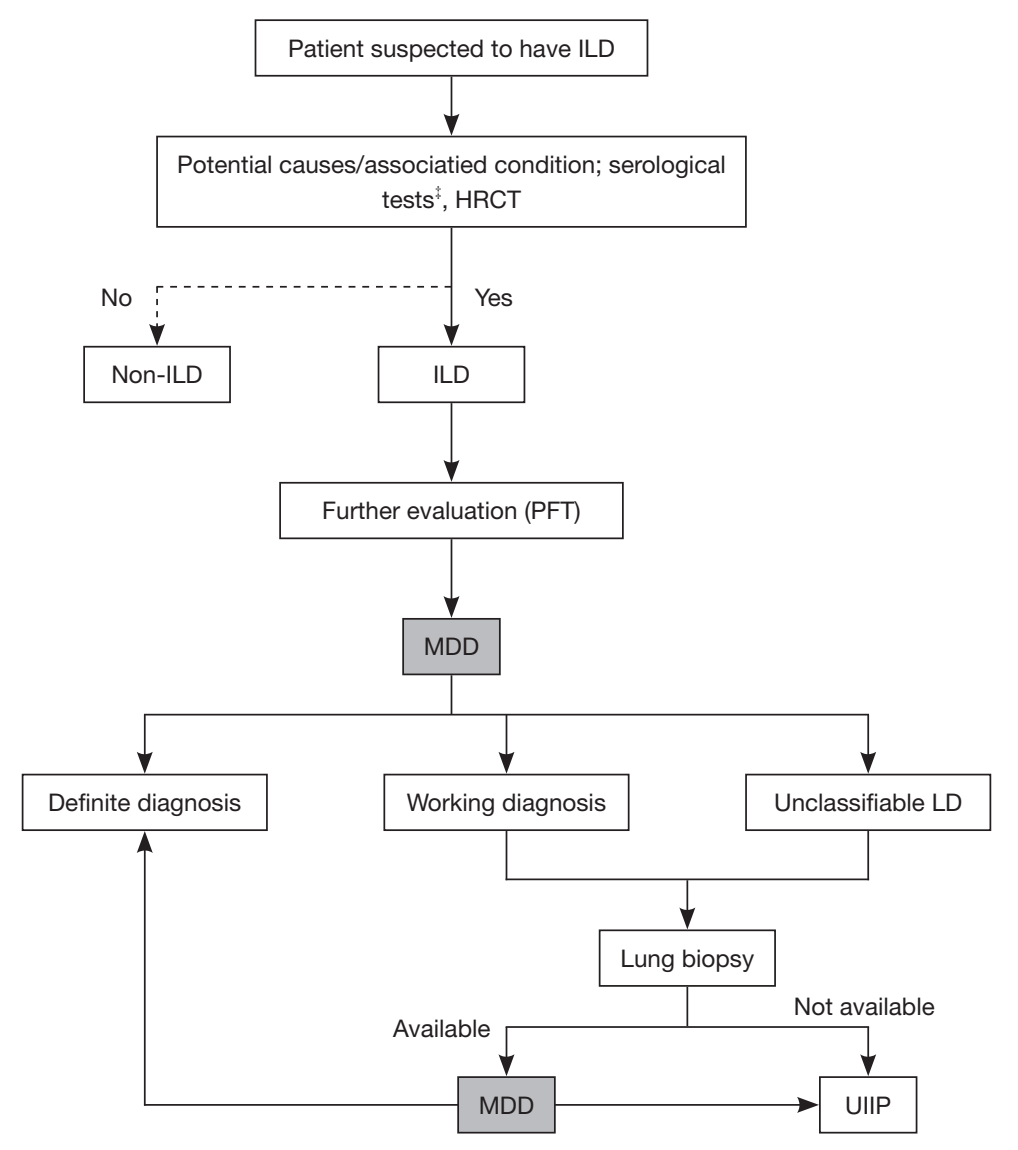

Figure 1 The diagnosis algorithm for ILDs. ILD, interstitial lung disease. ", Serological tests including antinuclear antibody (ANA), anticyclic citrullinated peptide antibody (CCP), anti-dsDNA, anti-Ro, anti-La, anti-ribonucleoprotein, anti-Smith, anti-topoisomerase, antitRNA synthetase(e.g., Jo-1, PL-7, PL-12, EJ, OJ), anti-PM-Scl, anti-MDA5, anti-rheumatoid factor (RF), erythrocyte sedimentation rate (ESR), C-reactive protein, and creatine phosphokinase.

or drug toxicity (see in the Supplementary). Second, all these patients would be ordered serological tests (antinuclear antibodies, rheumatoid factor, anti-cyclic citrulline antibody), chest high resolution computer tomography (HRCT), and pulmonary function test and diffusion capacity of the lung for carbon monoxide (DLco). Third, the first-round MDD was held by respiratory physicians and radiologists would made the diagnosis according to the aforementioned data. If working diagnosis and unclassifiable diagnosis of ILD were made after the first-round MDD, a lung biopsy would be considered after balancing the benefit and risk of different methods of biopsy. Before 2016, we usually performed transbronchial lung biopsy (TBLB) and SLB, and transbronchial cryobiopsy (TBCB) was adopted since 2016. The bronchoalveolar lavage was performed simultaneously with TBLB or TBCB for cell counting as well as to exclude infection. Finally, the second MDD including experienced respiratory physicians, radiologists, pathologists and rheumatologists (if CTD was suspected) was performed. MDD meeting was held face-to-face once a week in the meeting room routinely in our center. However, sometimes as to avoid delayed diagnosis, online meeting with other MDD members was carried out. The diagnosis algorithm for ILDs in our ILD center was shown in Figure 1.

\section{Clinical data and statistical analysis}

Clinical data including gender, age, diagnosis, forced vital capacity (FVC), DLco, HRCT, and pathology result were all collected. Clinical data were analyzed by using the SPSS 24.0 software package (SPSS Inc., Chicago, IL, USA). Normally distributed continuous variables were presented as the means \pm standard deviation (SD). Categorical variables were presented as constituent ratios. Descriptive statistics, i.e., frequencies and 
Table 1 The baseline characteristics of the ILD patients

\begin{tabular}{|c|c|}
\hline Characteristic & Value \\
\hline Age, years, (mean $\pm S D)$ & $57.9 \pm 13.4$ \\
\hline Male, n (\%) & $1,080(55.5)$ \\
\hline Smokers, n (\%) & $368(18.9)$ \\
\hline \multicolumn{2}{|l|}{ Symptom } \\
\hline Cough, n (\%) & $1,739(89.4)$ \\
\hline Dyspnea, n (\%) & $1,063(54.7)$ \\
\hline $\begin{array}{l}\text { Duration of symptoms at diagnosis } \\
\text { (month), median (IQR) }\end{array}$ & $8[3,25]$ \\
\hline \multicolumn{2}{|l|}{ Environmental exposure factor } \\
\hline Mold/mildew, n (\%) & $11(0.6)$ \\
\hline Farm, n (\%) & $7(0.4)$ \\
\hline Bird, n (\%) & $6(0.3)$ \\
\hline Humidifier, n (\%) & $3(0.2)$ \\
\hline Animals, n (\%) & $3(0.2)$ \\
\hline Wood, n (\%) & $3(0.2)$ \\
\hline Tint, n (\%) & $2(0.1)$ \\
\hline \multicolumn{2}{|l|}{ Occupational exposure factor } \\
\hline Silica, n (\%) & $5(0.3)$ \\
\hline Asbestos, $\mathrm{n}(\%)$ & $4(0.2)$ \\
\hline Coal, n (\%) & $2(0.1)$ \\
\hline Hard metal, n (\%) & $2(0.1)$ \\
\hline \multicolumn{2}{|l|}{ Pulmonary function test $(n=1,318)$} \\
\hline Normal, n (\%) & $317(24.1)$ \\
\hline Obstruction, n (\%) & $223(16.9)$ \\
\hline Mild, n (\%) & $128(57.4)$ \\
\hline Moderate, n (\%) & $61(27.4)$ \\
\hline Severe, n (\%) & $34(15.2)$ \\
\hline Restriction, n (\%) & $926(70.3)$ \\
\hline Mild, n (\%) & $462(49.9)$ \\
\hline Moderate, n (\%) & $318(34.3)$ \\
\hline Severe, n (\%) & $146(15.8)$ \\
\hline FVC \%predicted, (mean \pm SD) & $67.8 \pm 20.9$ \\
\hline \multicolumn{2}{|l|}{ Diffusion capacity of the lung $(n=1,227)$} \\
\hline Normal, n (\%) & $248(20.2)$ \\
\hline Mild, n (\%) & $425(34.6)$ \\
\hline Moderate, n (\%) & $368(30.0)$ \\
\hline Severe, n (\%) & $186(15.2)$ \\
\hline DLco \%predicted, (mean \pm SD) & $50.0 \pm 19.8$ \\
\hline
\end{tabular}

Table 1 (continued)
Table 1 (continued)

\begin{tabular}{lc}
\hline Characteristic & Value \\
\hline MDD & $1,945[100]$ \\
The first-round MDD, $n$ (\%) (including & \\
respiratory physicians and radiologists) & $818(42.1)$ \\
The second-round MDD, n (\%) (including & \\
respiratory physicians, radiologists, & \\
pathologists) & \\
\hline ILD, interstitial lung disease; FVC, forced vital capacity; \\
DLco, diffusion capacity of lungs for carbon monoxide; MDD, \\
multidisciplinary discussion.
\end{tabular}

percentages, were used to describe the study variables.

\section{Results}

A total of 1,945 individuals were diagnosed with ILDs in our ILD center from January 1, 2012, to December 31,2017 . The mean age (SD) was $57.9(13.4)$ years, and $1,080(55.5 \%)$ patients were male. The most common symptoms were cough $(89.4 \%)$ and dyspnea $(54.7 \%)$. There were $368(18.9 \%)$ patients presently or previously smoking. The median duration from symptoms onset to diagnosis was 8 months (IQR, 3-25). Only 23 (1.2\%) patients could provide the environmental and occupational exposure information in our study. The most common environmental factor was mold/mildew (11, 0.6\%), followed by farming $(7,0.4 \%)$, birds $(6,0.3 \%)$ and so on. The pulmonary function test and diffusion capacity were attempted in 1,318 patients. Among them, 926 (70.3\%) patients were suggestive of restrictive ventilation defection, $223(16.9 \%)$ patients were consistent with obstructive ventilation defection and 317 patients were normal. Among 1,227 patients who finished DLco measurements (corrected for Hemoglobin), DLco was decreased in 979 (79.8\%) patients. The mean (SD) percentage of the FVC and DLco of the predicted value were 67.8 (20.9) and 50.0 (19.8), respectively. The first-round MDD was held in all patients and the second-round MDD including respiratory physicians, radiologists and pathologists was held in $818(42.1 \%)$ patients. The details of baseline characteristics of the ILD patients were shown in Table 1.

In generally, IPF $(395,20.3 \%)$ was the most frequent subgroup of ILDs, followed by CTD-ILD (356, 18.3\%), IPAF $(349,17.9 \%)$, and UIIP $(285,14.7 \%)$. The spectrum and clinical characteristic of ILDs was shown in Table 2. Among the CTD-ILD patients, the mean age (SD) was 
Table 2 The Spectrum and Clinical Characteristic of ILDs

\begin{tabular}{|c|c|c|c|c|c|}
\hline ILD & Patients, No. $\left(\%{ }^{*}\right)$ & Age (mean $\pm S D)$, y & $\begin{array}{l}\text { Gender ratio } \\
\text { (man/woman) }\end{array}$ & $\begin{array}{l}\text { FVC\%pred } \\
\text { (mean } \pm \text { SD) }\end{array}$ & $\begin{array}{l}\text { DLco\%pred } \\
\text { (mean } \pm \text { SD) }\end{array}$ \\
\hline ILD of known causes & $382(19.6)$ & $55.5 \pm 12.5$ & $1: 1.6$ & $67.4 \pm 19.9$ & $50.5 \pm 17.7$ \\
\hline CTD-ILD & $356(18.3)$ & $56.1 \pm 12.3$ & $1: 1.5$ & $67.4 \pm 19.8$ & $50.1 \pm 17.6$ \\
\hline RA-ILD & $116(6.0)$ & $58.7 \pm 11.7$ & $1: 1.2$ & $69.0 \pm 9.42$ & $45.7 \pm 7.1$ \\
\hline pSS-ILD & $50(2.6)$ & $59.1 \pm 11.0$ & $1: 2.6$ & $74.4 \pm 19.9$ & $57.2 \pm 17.3$ \\
\hline SS-C-ILD & $49(2.5)$ & $52.1 \pm 10.1$ & $1: 6$ & $62.9 \pm 17.8$ & $49.7 \pm 16.7$ \\
\hline Vasculitis--ILD & $33(1.7)$ & $54.5 \pm 16.5$ & $1: 0.6$ & $80.1 \pm 22.7$ & $56.5 \pm 18.1$ \\
\hline MCTD-ILD & $15(0.8)$ & $55.3 \pm 11.2$ & $1: 4$ & $53.7 \pm 10.3$ & $70.7 \pm 9.0$ \\
\hline Occupation & $13(0.7)$ & $42.8 \pm 9.4$ & $1: 1.6$ & $62.9 \pm 20.1$ & $58.7 \pm 23.8$ \\
\hline IIP & $784(40.3)$ & $62.3 \pm 11.4$ & $1: 0.3$ & $62.0 \pm 26.1$ & $44.1 \pm 18.1$ \\
\hline IPF & 395 (20.3) & $65.3 \pm 9.8$ & $1: 0.1$ & $63.5 \pm 26.9$ & $41.5 \pm 16.3$ \\
\hline UIIP & $285(14.7)$ & $61.5 \pm 9.8$ & $1: 0.7$ & $58.1 \pm 24.6$ & $44.3 \pm 18.0$ \\
\hline NSIP & $53(2.7)$ & $54.0 \pm 11.7$ & $1: 0.9$ & $67.3 \pm 20.5$ & $56.9 \pm 19.5$ \\
\hline COP & $26(1.3)$ & $53.4 \pm \pm 9.2$ & $1: 2.3$ & $77.8 \pm 20.4$ & $54.2 \pm 17.4$ \\
\hline LIP & $10(0.5)$ & $48.9 \pm 13.1$ & $1: 1.5$ & $77.5 \pm 30.5$ & $66.9 \pm 23.7$ \\
\hline Rare & $248(12.8)$ & $40.4 \pm 14.1$ & $1: 1.5$ & $79.5 \pm 20.5$ & $57.9 \pm 21.9$ \\
\hline PLCH & $21(1.1)$ & $29.4 \pm 8.7$ & $1: 0.2$ & $75.9 \pm 18.1$ & $50.4 \pm 9.8$ \\
\hline PAP & $121(6.2)$ & $45.0 \pm 10.8$ & $1: 0.9$ & $77.7 \pm 15.9$ & $60.1 \pm 20.3$ \\
\hline LAM & 67 (3.4) & $39.8 \pm 10.2$ & $0: 67$ & $85.6 \pm 29.1$ & $48.8 \pm 22.7$ \\
\hline Others & $39(2.0)$ & $38.4 \pm 17.5$ & $1: 1.2$ & $77.5 \pm 20.1$ & $70.0 \pm 23,9$ \\
\hline IPAF & 349 (17.9) & $58.4 \pm 12.6$ & $1: 1.7$ & $69.1 \pm 19.6$ & $53.3 \pm 15.9$ \\
\hline TOTAL & $1,945[100]$ & $57.9 \pm 13.4$ & $1: 0.8$ & $67.8 \pm 20.9$ & $50.0 \pm 19.8$ \\
\hline
\end{tabular}

*, proportion of ILD entity in all the ILD patients. ILD, interstitial lung disease; CTD, connective tissue disease; RA, rheumatoid arthritis; IIM, idiopathic inflammation myositis; SS-c, systemic sclerosis; pSS, primary Sjogren's syndrome; MCTD, mixed connective tissue disease; SLE, systemic Lupus Erythematosus; IIP, idiopathic interstitial pneumonia; IPF, idiopathic pulmonary fibrosis; UIIP, unclassifiable idiopathic interstitial pneumonia; NSIP, idiopathic nonspecific interstitial pneumonia; COP, cryptogenic organizing pneumonia; LIP, idiopathic lymphoid interstitial pneumonia; AIP, acute interstitial pneumonia; RB-ILD, respiratory bronchiolitis-interstitial lung disease; DIP, desquamative interstitial pneumonia; HP, hypersensitivity pneumonitis; PLCH, Pulmonary Langerhans cell histiocytosis; PAP, pulmonary alveolar proteinosis; LAM, pulmonary lymphangiomyomatosis; IPAF, interstitial pneumonia with autoimmune features. 
Table 3 the Histological diagnosis of ILDs patients

\begin{tabular}{|c|c|c|c|c|}
\hline \multirow{2}{*}{$\begin{array}{l}\text { Biopsy } \\
\text { methods }\end{array}$} & \multicolumn{2}{|c|}{ Clinically helpful diagnoses } & \multirow{2}{*}{$\begin{array}{c}\text { No } \\
\text { diagnoses }\end{array}$} & \multirow[b]{2}{*}{ Total } \\
\hline & $\begin{array}{l}\text { Definitive } \\
\text { diagnoses }\end{array}$ & $\begin{array}{c}\text { Working } \\
\text { diagnoses }\end{array}$ & & \\
\hline TBLB & $115(20.8)$ & $345(62.3)$ & $94(16.9)$ & 554 \\
\hline SLB & $189(93.6)$ & $13(6.4)$ & 0 & 202 \\
\hline $\begin{array}{l}\text { TBLB and } \\
\text { SLB }\end{array}$ & $11(100.0)$ & 0 & 0 & 11 \\
\hline TBCB & $39(76.5)$ & $10(19.6)$ & $2(3.9)$ & 51 \\
\hline
\end{tabular}

ILD, interstitial lung disease; TBLB, transbronchial lung biopsy; SLB, surgical lung biopsy; TBCB, transbronchial cryobiopsy.

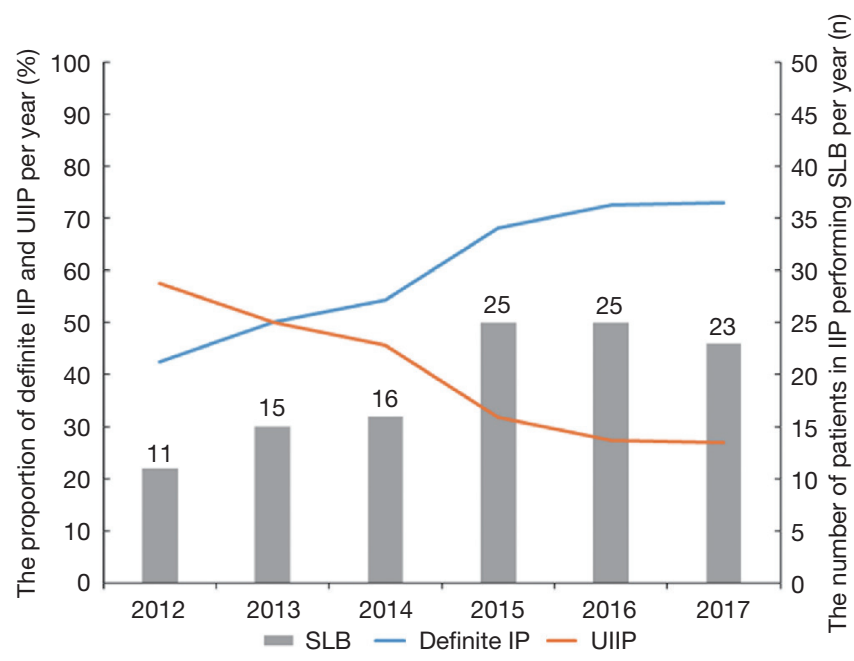

Figure 2 The spectrum of IIP and the number of SLB cases per year. IIP, idiopathic interstitial pneumonia; SLB, surgical lung biopsy.

56.1 (12.3), and there was a predominance of female (214, $60.1 \%)$. The most frequent subgroup of CTD-ILD was rheumatoid arthritis (RA)-ILD (116, 32.6\%), followed by Polymyositis/Dermatomyositis PM/DM-ILD (89, 25.0\%), primary Sjogren's syndrome (pSS)-ILD (50, 14.0\%). In patients diagnosed with IPAF, the mean age (SD) was 58.4 (12.6), and the male-to-female ratio was 1:1.7.

In IIP subgroups, the mean age (SD) was 62.3 (11.4), and the male-to-female ratio was 1:0.3. The mean (SD) percentage of the FVC and DLco of the predicted value were 62.0 (20.1) and 44.1 (18.1), respectively. In the IIP subgroup, IPF $(395,50.4 \%)$ took the highest proportion, followed by UIIP $(285,36.4 \%)$, nonspecific interstitial pneumonia (NSIP) $(53,6.8 \%)$ and cryptogenic organizing pneumonia (COP) $(26,3.3 \%)$.

In patients diagnosed with HP, there were 35 (59.3\%) patients processing definite environmental exposure, among whom thirty-two patients were diagnosed according to typical HRCT features and three were diagnosed after SLB, since their HRCT showed probably usual interstitial pneumonia (UIP) pattern. Moreover, there were twentyfour $(40.7 \%)$ patients without known exposure diagnosed as HP based on typical HRCT features and SLB.

There were 818 (42.1\%) patients underwent lung biopsy to obtain a histological diagnose. TBLB was performed in $565(29.0 \%)$ patients, eleven of whom underwent SLB because TBLB failed to obtain lung samples. SLB was performed in $213(11.0 \%)$ patients and TBCB was performed in $51(2.6 \%)$ patients. Specimens were considered clinically helpful in $815(89.3 \%)$ patients, in which definitive histopathological diagnoses were provided in 436 cases. Compared to TBLB, SLB and TBCB contributed more cases of definitive diagnoses in MDD. The details of histological diagnoses based on different lung biopsy methods was shown in Table 3.

In the subgroup of IIP, we defined patients diagnosed with definite IIP in order to distinguish them from UIIP. Definite IIP included IPF, NSIP, COP, lymphoid interstitial pneumonia (LIP), acute interstitial pneumonia (AIP), respiratory bronchiolitis-interstitial lung disease (RB-ILD) and desquamative interstitial pneumonia (DIP). From 2012 to 2017 , the proportions of definite IIP increased from $42.5 \%$ to $73.0 \%$ per year, while the proportion of UIIP decreased from $57.5 \%$ to $27.0 \%$ per year. A total $115(29.7 \%)$ individuals underwent SLB for pathologic diagnosis. In patient diagnosed with IIP, SLB accounted for the highest proportion of biopsies in LIP $(10 / 10,100 \%)$ and RB-ILD/DIP (9/9, 100\%) cases, followed by COP (18/26, $69.2 \%)$, NSIP $(22 / 53,41.5 \%)$ and IPF $(43 / 395,10.9 \%)$. However, thirteen individuals (11.3\%) still diagnosed as UIIP because of the conflict of radiological and pathological information. From 2012 to 2017, the number of patient diagnosed with IIP underwent SLB each year increased from 11 to 25/year. The spectrum of IIP and the number of SLB cases per year were shown in Figure 2.

\section{Discussion}

This retrospective study revealed that IPF was the most common subgroup of ILDs, followed by IPAF and CTDILD in our center.

The proportion of IPF in our center was similar to 
other studies, varying from $20.1 \%$ to $26.5 \%(9,13,23)$, but was lower than that of other studies reported prior to 2002 , which showed rates varying from $32.5 \%$ to $38.6 \%$ $(24,25)$. It could be explained by our strict application of 2011 and 2018 ATS/ERS/JRS/ALAT diagnostic criteria $(15,26)$. Many cases previously labelled as IPF before 2002 are now reclassified as fibrosis NSIP, ILDs secondary to CTDs with UIP pattern in radiological or pneumoconiosis. In our center, the proportion of UIIP was $14.7 \%$, and was similar to other cohort studies, varying from $1.8 \%$ to $15.4 \%$ $(7,9,23,25,27)$. Although SLB is essential in the diagnosis and classification of IIPs, there were still large number of patients $(158 / 285,58.1 \%)$ who had high risk in SLB (data was not shown). There were also13 (4.6\%) patients who received SLB, but showed a conflict among clinical, radiological and pathological information, they were finally diagnosed with UIIP after the second MDD.

Notably, we found that the proportion of CTDILD and IPAF are $36.2 \%$, a proportion even larger than that of IPF. Both CTD-ILD and IPAF are driven by autoimmune processes and could be treated by steroids and immunosuppressants. IPAF includes patients with IIP features and underlying autoimmune diseases but does not meet the established criteria for CTD (14). In previous published studies, CTD-ILD represent $9.8-16.0 \%$ of ILDs $(7,8,28)$. Our result of higher proportion may be related to our awareness of the CTDs signs and autoimmune serologies and MDD involving rheumatologists routinely. We found that in CTD-ILD patients, the most frequent immune disease was RA $(116,32.6 \%)$, followed by idiopathic inflammation myositis (IIM) (89, 25.0\%), primary Sjogren's syndrome (pSS) $(50,14.0 \%)$ and systemic sclerosis (SSc) (49, 13.8\%). The IIM-ILD, SSc-ILD, IPAF and pSS-ILD groups had stronger female predominant. In contract to RA-ILD patients, patients were older and gender balance. This result was similar to another study reported the prevalence of ILDs in other ILD center in China (13).

The proportion of HP varies from $2.7-47.3 \%$ of ILDs in other cohort $(7,8,12,13,25)$. One study from India reported that the proportion of HP reached $47.3 \%$ because of the environmental/local cultural factors, in which air conditioner accounted for the highest proportion (12). In Singh's study, the high proportion of HP may be attributable to their case report forms designed specifically for unmasking exposures to HP. However, the cause and effect on the cooler pads and HP was unclear. However, we found only $3.0 \%$ of ILDs diagnosed as HP in our center, although we have performed relatively complete screening of environmental factors. Actually, chronic HP is difficult to diagnose due to the obscure exposure history, unknown causative antigen, lack of serum $\operatorname{IgG}$ against various microbial and avian antigens (29) and low agreement across MDD on diagnosis (30).

The quality of pulmonary histology is crucial for histological diagnosis in some selected patients. TBLB could be useful in some selected ILDs. We found that the diagnostic yield TBLB in PLAM, PAP and sarcoidosis were 95.5\%, $96.7 \%$ and $97.6 \%$, respectively (data not shown). Our previous study reported that TBLB could only useful for $30.4 \%$ of ILD patients, of whom the majority were finally diagnosed with PAP (31).

SLB was considered as the current gold standard for obtaining adequate lung biopsy specimens (15). In our study, 213 (11.0\%) individuals underwent SLB after the first round MDD with 200 (93.9\%) of them finally specially. In the IIP subgroup, we found that the proportion of definite IIP gradually increased, which may be related with the increased proportion of SLB and the implementation of TBCB. On the other hand, TBCB was suggested as the alternative of SLB in the diagnosis of lesions with bronchovascular or peri-lymphatic distribution (32), although the consistency of TBCB and SLB in the ILD diagnosis was still controversy and needs further exploration (33). Unfortunately, the performance of TBCB was after 2016 with few patients, thus we cannot compare the two biopsies during this stage.

Albeit the importance of histological information in the diagnosis of ILDs, we must remain in mind that all the biopsies may be associated with certain morbidity and mortality. Therefore the pre-operative evaluation is as important as, if not more important than, the sophisticated skills (34). It was recommended that two-round MDD should be held during the ILD diagnosis, with the first round for biopsy evaluation (26). We followed this diagnostic algorithm in this study in an attempt to balance the specific diagnosis and biopsy risk.

There are several limitations to this study. First, it was a retrospective study conducted in a single center. Meanwhile, as the largest ILD center in southern China, the results in our institute may reflect the spectrum of ILDs in the southern region. Second, we could not know the rate of change in the medical therapy of patients underwent SLB. Since the post-operative mortality rates ranged from $3 \%$ to $16.7 \%$ in previous study (34). We should do more research to find out whether SLB could 
result in change in the treatment and even outcome of ILDs patients.

\section{Conclusions}

In summary, IPF is the most frequent ILDs in southern China, followed by CTD-ILD and IPAF. When clinical and radiological information is too limited to make a confident diagnosis, a histological information may serve as a key determinants or supplementary information in the final diagnosis.

\section{Acknowledgments}

Funding: This work is supported by National Key Research Project-Accurate Medical Research (No. 2016YFC0905701) and "Foundation for Young Scholars of Guangzhou Medical University".

\section{Footnote}

Data Sharing Statement: Available at http://dx.doi. org/10.21037/jtd-19-4021

Conflicts of Interest: All authors have completed the ICMJE uniform disclosure form (available at http://dx.doi. org/10.21037/jtd-19-4021). The authors have no conflicts of interest to declare.

Ethical Statement: The authors are accountable for all aspects of the work in ensuring that questions related to the accuracy or integrity of any part of the work are appropriately investigated and resolved. The trial was conducted in accordance with the Declaration of Helsinki (as revised in 2013) and the Harmonized Tripartite Guideline for Good Clinical Practice from the International Conference on Harmonization. The study was approved by the Institutional Human Ethics Review Committee in our institute (approval number $2020 \mathrm{~K}-10$ ). All patients enrolled completed the informed consent form.

Open Access Statement: This is an Open Access article distributed in accordance with the Creative Commons Attribution-NonCommercial-NoDerivs 4.0 International License (CC BY-NC-ND 4.0), which permits the noncommercial replication and distribution of the article with the strict proviso that no changes or edits are made and the original work is properly cited (including links to both the formal publication through the relevant DOI and the license). See: https://creativecommons.org/licenses/by-nc-nd/4.0/.

\section{References}

1. Raghu G, Collard HR, Egan JJ, et al. An official ATS/ ERS/JRS/ALAT statement: idiopathic pulmonary fibrosis: evidence-based guidelines for diagnosis and management. Am J Respir Crit Care Med 2011;183:788-824.

2 Walsh SL, Calandriello L, Sverzellati N, et al. Interobserver agreement for the ATS/ERS/JRS/ALAT criteria for a UIP pattern on CT. THORAX 2016;71:45-51.

3 Nicholson AG, Addis BJ, Bharucha H, et al. Inter-observer variation between pathologists in diffuse parenchymal lung disease. THORAX 2004;59:500-5.

4 American Thoracic Society/European Respiratory Society International Multidisciplinary Consensus Classification of the Idiopathic Interstitial Pneumonias. This joint statement of the American Thoracic Society (ATS), and the European Respiratory Society (ERS) was adopted by the ATS board of directors, June 2001 and by the ERS Executive Committee, June 2001. Am J Respir Crit Care Med 2002;165:277-304.

5 Lynch DA, Sverzellati N, Travis WD, et al. Diagnostic criteria for idiopathic pulmonary fibrosis: a Fleischner Society White Paper. Lancet Respir Med 2018;6:138-53.

6 Grewal JS, Morisset J, Fisher JH, et al. Role of a Regional Multidisciplinary Conference in the Diagnosis of Interstitial Lung Disease. Ann Am Thorac Soc 2019; 16:455-62.

7 Hyldgaard C, Hilberg O, Muller A, et al. A cohort study of interstitial lung diseases in central Denmark. Respir Med 2014;108:793-9.

8 Musellim B, Okumus G, Uzaslan E, et al. Epidemiology and distribution of interstitial lung diseases in Turkey. Clin Respir J 2014;8:55-62.

9 Karakatsani A, Papakosta D, Rapti A, et al. Epidemiology of interstitial lung diseases in Greece. Respir Med 2009;103:1122-9.

10 Coultas DB, Zumwalt RE, Black WC, et al. The epidemiology of interstitial lung diseases. Am J Respir Crit Care Med 1994;150:967-72.

11 Dhooria S, Agarwal R, Sehgal IS, et al. Spectrum of interstitial lung diseases at a tertiary center in a developing country: A study of 803 subjects. PLoS One 2018;13:e0191938.

12 Singh S, Collins BF, Sharma BB, et al. Interstitial Lung Disease in India. Results of a Prospective Registry. Am J 
Respir Crit Care Med 2017;195:801-13.

13 Ban C, Yan W, Xie B, et al. Spectrum of interstitial lung disease in China from 2000 to 2012. Eur Respir J 2018;52:1701554.

14 Fischer A, Antoniou KM, Brown KK, et al. An official European Respiratory Society/American Thoracic Society research statement: interstitial pneumonia with autoimmune features. Eur Respir J 2015;46:976-87.

15 Travis WD, Costabel U, Hansell DM, et al. An official American Thoracic Society/European Respiratory Society statement: Update of the international multidisciplinary classification of the idiopathic interstitial pneumonias. Am J Respir Crit Care Med 2013;188:733-48.

16 Arnett FC, Edworthy SM, Bloch DA, et al. The American Rheumatism Association 1987 revised criteria for the classification of rheumatoid arthritis. Arthritis Rheum 1988;31:315-24.

17 Preliminary criteria for the classification of systemic sclerosis (scleroderma). Subcommittee for scleroderma criteria of the American Rheumatism Association Diagnostic and Therapeutic Criteria Committee. Arthritis Rheum 1980;23:581-90.

18 Tan EM, Cohen AS, Fries JF, et al. The 1982 revised criteria for the classification of systemic lupus erythematosus. Arthritis Rheum 1982;25:1271-7.

19 Smolen JS, Steiner G. Mixed connective tissue disease: to be or not to be? Arthritis Rheum 1998;41:768-77.

20 Bohan A, Peter JB. Polymyositis and dermatomyositis (first of two parts). N Engl J Med 1975;292:344-7.

21 Lacasse Y, Selman M, Costabel U, et al. Clinical diagnosis of hypersensitivity pneumonitis. Am J Respir Crit Care Med 2003;168:952-8.

22 Statement on sarcoidosis. Joint Statement of the American Thoracic Society (ATS), the European Respiratory Society (ERS) and the World Association of Sarcoidosis and Other Granulomatous Disorders (WASOG) adopted by the ATS Board of Directors and by the ERS Executive Committee, February 1999. Am J Respir Crit Care Med 1999;160:736-55.

23 Alhamad EH. Interstitial lung diseases in Saudi Arabia: A single-center study. Ann Thorac Med 2013;8:33-7.

Cite this article as: Guo B, Wang L, Xia S, Mao M, Qian W, Peng X, Zheng Z, Chen R, Han Q, Luo Q. The interstitial lung disease spectrum under a uniform diagnostic algorithm: a retrospective study of 1,945 individuals. J Thorac Dis 2020;12(7):3688-3696. doi: 10.21037/jtd-19-4021
24 Schweisfurth H. Report by the Scientific Working Group for Therapy of Lung Diseases: German Fibrosis Register with initial results. Pneumologie 1996;50:899-901.

25 Xaubet A, Ancochea J, Morell F, et al. Report on the incidence of interstitial lung diseases in Spain. Sarcoidosis Vasc Diffuse Lung Dis 2004;21:64-70.

26 Raghu G, Remy-Jardin M, Myers JL, et al. Diagnosis of Idiopathic Pulmonary Fibrosis. An Official ATS/ERS/JRS/ ALAT Clinical Practice Guideline. Am J Respir Crit Care Med 2018;198:e44-68.

27 Storme M, Semionov A, Assayag D, et al. Estimating the incidence of interstitial lung diseases in the Cree of Eeyou Istchee, northern Quebec. PLoS One 2017;12:e0184548.

28 Duchemann B, Annesi-Maesano I, acobe de Naurois C, et al. Prevalence and incidence of interstitial lung diseases in a multi-ethnic county of Greater Paris. Eur Respir J 2017;50:1602419.

29 Vasakova M, Morell F, Walsh S, et al. Hypersensitivity Pneumonitis: Perspectives in Diagnosis and Management. Am J Respir Crit Care Med 2017;196:680-9.

30 Walsh SL, Wells AU, Desai SR, et al. Multicentre evaluation of multidisciplinary team meeting agreement on diagnosis in diffuse parenchymal lung disease: a casecohort study. Lancet Respir Med 2016;4:557-65.

31 Han Q, Luo Q, Chen X, et al. The evaluation of clinical usefulness of transbrochoscopic lung biopsy in undefined interstitial lung diseases: a retrospective study. Clin Respir J 2017;11:168-75.

32 Hetzel J, Maldonado F, Ravaglia C, et al. Transbronchial Cryobiopsies for the Diagnosis of Diffuse Parenchymal Lung Diseases: Expert Statement from the Cryobiopsy Working Group on Safety and Utility and a Call for Standardization of the Procedure. Respiration 2018;95:188-200.

33 Troy LK, Grainge C, Corte TJ, et al. Diagnostic accuracy of transbronchial lung cryobiopsy for interstitial lung disease diagnosis (COLDICE): a prospective, comparative study. Lancet Respir Med 2020;8:171-81.

34 Fisher JH, Shapera S, To T, et al. Procedure volume and mortality after surgical lung biopsy in interstitial lung disease. Eur Respir J 2019;53:1801164. 
1. The Detailed History of Patients with ILDs

1.1 Family History (grandparents, parents, brothers, sisters, aunts, uncles, cousins, or children)

$\square$ Pulmonary Fibrosis

$\square$ Connective Tissue Disease

$\square$ Sarcoidosis

$\square$ Hypersensitivity Pneumonitis

\subsection{Environmental or Occupational Exposure History (if}

the answer is "yes", please providing the exposure time)

$\square$ Bird (Include Pigeons, Doves, Parakeets, Chickens, Ducks, Geese, Pheasants)

$\square$ Animals (Dogs, Cats, Rabbits, Gerbils, Hamsters)

$\square$ Humidifier

$\square$ Mold/Mildew

$\square$ Potting Soil (Plants)

$\square$ Carpet

$\square$ Foundry

$\square$ Smelting

$\square$ Railroad

$\square$ Tint

$\square$ Plastic Factory $\square$ Metal

$\square$ Silica

$\square$ Asbestos

$\square$ Cotton

$\square$ Wood

$\square$ Paint

1.3 History of signs associated with connective tissue disease (if the answer is "yes", please providing the exposure time)

$\square$ Difficulty swallowing

$\square$ Heartburn, reflux, or sour taste in mouth after eating

$\square$ Persistently dry eyes or dry mouth

$\square$ Rash or change in skin

$\square$ Foot or leg swelling

$\square$ Sensitivity to light

$\square$ Hand ulcers

$\square$ Alopecia

$\square$ Dental caries

$\square$ Mouth ulcers

$\square$ Ulcers in vagina

$\square$ Chest pain

$\square$ Joint stiffness, pain or swelling 